Ville et campagne

dans l'île d'Iviça

Joan Vilà i Valentí

Territoris (2000), 3 :

205-218 


\section{Ville et campagne dans l'île d'Iviça}

JOAN VILÀ VALENTÍ (1962) : Ville et campagne dans l'île d'Eivissa. In "Mediterranée". Université d’Aix-enProvence. III, 4. 51 - 65. Hi ha una versió al castellà de B. Barceló Pons: "Ciudad y Campo en la Isla de Ibiza", Palma, BCOCINP, 1963, T. LXV, n. 639, pp. 94-101. Aquesta versió va ser reproduïda a "Arquitectura y espacio rural en Ibiza. Publicación de la Delegación en Ibiza y Formentera del Colegio de Arquitectos de Baleares". Eivissa, 1981-1982, núm. 4-5. 16-23. Aquesta derrera edició du una referència biogràfica de J. Vilà Valentí i una presentació del mateix autor, però no inclou la bibliografia de l'original.

\section{SUMARI}

\section{INTRODUCTION}

\section{L'OPPOSITION ENTRE VILLE ET CAMPAGNE}

\section{Les différences.}

L'opposition violente.

II. LES RELATIONS TRADITIONNELLES

La ville traditionnelle.

La fonction religieuse.

La fonction militaire.

Les fonctions politiques et administratives

Les fonctions économiques

Le port.

\section{L'ÉVOLUTION RÉCENTE}

L'évolution de la ville.

L'évolution de la campagne.

Situation actuelle et perspectives.

Nous avons parlé à diverses reprises des différences géographiques marquées, physiques et humaines, que présentent entre elles les îles Baléares, en dépit de certaines caractéristiques communes. La personnalité, bien déterminée, de chacune de ces îles apparait à nouveau nettement si I'on considère les rapports qui se sont établis, dans chacune d'entre elles, entre la campagne, d'une part, et les noyaux urbains, d'autre part. C'est là un problème qui intéresse vivement à sociologues et géographes urbains et que nous voudrions étudier, dans ce travail, pour la seule île d'Iviça.

Le cas le plus complexe, au sein des Baléares, est évidemment celui de Majorque. En dépit de la forte influence que la ville de Palma exerce sur toute l'île, d'autres noyaux urbains y sont apparus depuis longtemps, constituant des sortes de centres secondaires qui donnent naissance à un réseau compliqué de rapports entre l'aire majorquine et sa capitale. 
Ainsi, Palma n'a pas le monopole des fonctions urbaines et n'exerce pas directement son influence sur toute la superficie insulaire. Beaucoup plus simple est le cas de Minorque; cependant, là encore deux noyaux urbains bien différenciés apparaissent chacun avec ses propres fonctions.

Par contre, Formentera manque de centre urbain, comme nous avons déjà montré ailleurs (VILÀ VALENTÍ,1950; voir la référence complète dans la bibliographie citée à la fin de cet article). En réalité, cette île peut parfaitement être considérée, à notre point de vue, comme un prolongement de la campagne d'Iviça.

Le cas d'Iviça s'oppose à la fois à celui de I'une et l'autre des deux Iles Baléares restantes. Cette île a toujours présenté un seul noyau urbain, dont I'influence s'exerce de telle sorte qu'elle s'étend sur Iviça tout entière, sans qu'il existe aucun autre centre intermédiaire. On ne pourrait trouver d'exemple aussi parfaitement caractéristique. Pendant des siècles, I'île d'Iviça a été exactement ceci: une ville et une campagne.

\section{L'opposition entre ville et campagne}

Les différences très marquées qui existent entre ville et campagne n'échappent à personne, particulièrement à Iviça. Aussi, encore que cela puisse paraître quelque peu paradoxal, avant de parler des rapports proprement dits entre ville et campagne, nous présenterons brièvement les oppositions qui existent entre celle-ci.

\section{Les différences}

Des notables différences apparaissent, à tous les points de vue. En premier lieu, en ce qui concerne le paysage: d'un côté, la ville, en acropole, nettement limitée par ses murailles (la Vila); d'un autre côté, la vaste campagne, avec ses cultures céréalières et arboricoles, son habitat dispersé, ses petites collines (puigs) avec leurs pinèdes, jusqu'aux confins insulaires.

Opposition économique, en second lieu, au sujet de laquelle nous insisterons plus loin, entre le commerce et l'artisanat de la vila, d'une part, et l'agriculture, l'élevage et l'exploitation forestière, campagnards, d'autre part; opposition sociale, aussi, dans les genres de vie entre les propriétaires fonciers et la bourgeoisie vivant dans la ville, surtout dans la haute ville (Dalt Vila) et le cultivateur de la campagne (es pagès). Opposition, enfin, entre les fonctions complexes de la concentration urbaine et les activités exclusivement rurales du reste de I'île.

Il n'y a pas longtemps, un auteur insulaire insistait sur les différences existentes entre le parler de la ville face à celui de la campagne (VILLANGÓMEZ, 1957). Il s'agit là évidemment de la projection matérielle, dans quelque chose d'aussi vivant que le langage, de l'existence des deux communautés distinctes, I'une urbaine, l'autre rurale. On pourrait facilement étudier d'une façon plus poussée d'autres aspects culturels -certaines coutumes, la mentalité elle-méme- afin de préciser les contrastes, également marqués dans ces domaines, entre les deux groupes humains.

\section{L'opposition violente}

Les divergences entre ville et campagne sont parfois si accusées qu'elles arrivent á s'exprimer sous une forme trés contrastée, même violente: l'hégémonie que le noyau urbain iviçain est arrivé á détenir, en particular à certaines époques, a donné lieu dans les campagnes à un état de ressentiment permanent qui a pu parfois prendre même la forme 
d'un mouvement armé. L'exposition des faits qui fut faite au roi en 1690 par Joan Sunyer, au nom des syndics et députés des «parties foraines», est très intéressante à cet égard (MACABICH, 1942).

Une étude de ces manifestations serait fort intéressante, que seule une analyse exhaustive des archives d'Iviça pourrait peu-être rendre possible. Les documents connus permettent de suivre des mouvements armés de pagesos (paysans) contre la ville depuis le XVème siècle au moins. Ils ont constitué un aspect des crises économiques et sociales de ce siècle et présentent selon, toute vraisemblance, des caractères semblables à ceux qu'ont revêtu les luttes qui eurent lieu à la même époque dans I'île voisine de Majorque entre les urbains et les gens de la campagne, appelés forans, c'est-à-dire les extérieurs ou forains (QUADRADO,1895). Au cours du siècle dernier, des émeutes se produisirent encore à diverses reprises pendant les quelles s'affrontirent des groupes de paysans et les gens de la ville.

Ces faits ne peuvent surprendre puisque, dans des circonstances plus ou moins semblables, ils se sont produits pendant ces siècles dans d'autres régions de l'Europe occidentale. La vie était très dure pour les paysans, soumis á de nombreuses charges sociales et économiques, ayant un niveau de vie très bas, et décimés périodiquement par la peste et par la faim. Ainsi, comme il ne fait pas de doute que ces plaies affligèrent également notre île, le soulèvement de la campagne constitue une manifestation de révolte contre la ville et le patriciat urbain qui la dominait, oligarchie n'oeuvrant presque toujours, à l'exception de quelques cas, que dans son strict intérêt.

\section{Les relations traditionelles}

Cependant, en dépit des oppositions que nous venons de montrer, souvent si éclatantes, des liens profonds existent entre la ville d'Iviça et l'ensemble de I'île, liens qui peuvent paraître parfois subtils, mais toujours variés et étroits, en particulier, à ce qu'on en sait, après la Reconquête, date à partir de laquelle l'abondante documentation existante nous permet de brosser un tableau complet de l'influence de la ville sur toute l'aire insulaire. Il est possible qu'à d'autres époques la situation ait été semblable. Nous pensons par exemple à la période punico-romaine, quand le noyau d'Iviça fut probablement aussi le seul centre urbain de I'île. Mais les sources archéologiques et littéraires (Strabon, Diodore de Sicile, etc.) ne nous permettent pas d'avoir une idée précise du problème qui nous intéresse ici. Nous nous bornerons donc à parler de la période que l'on pourrait appeler traditionnelle, et dont la durée s'étend au moins sur cinq siècles, du XIII ème. au XVIII ème.

\section{La ville traditionnelle}

Il convient de préciser, d'abord, ce que nous entendons par «ville» pendant la période traditionnelle. En effet, on doit tenir compte du fait que ce concept ne correspond pas exactement à celui de noyau urbain dans l'époque actuelle, ce noyau qui est né ou s'est développé sous l'impulsion de la révolution industrielle du siècle passé. Nous envisageons, au contraire, une ville d'origine médiévale qui présente, comme il est fréquent dans la région méditerranéenne, de nombreuses ressemblances avec les centres urbains de la période antique. Il faut aussi insister sur le fait qu'il n'existe pas seulement une différence quantitative entre l'agglomération médiévale et la ville actuelle, différences qui consisterait en une supériorité du seul nombre de ses habitants. Il existe aussi des différences qualitatives, résultat du phénomène économique auquel il a été précédemment fait allusion et qu'il n'est pas question de traiter ici. 
Iviça a constituée, pendant la période traditionnelle, un parfait exemple de ville médiévale. Il s'agit d'un noyau urbain avec des fonctions religieuses, militaires, politiques, administratives, artisanales et commerciales, environné de sa campagne. Cette campagne, de laquelle il est séparé nettement et totalement par les murailles, constitue le pays qui nourrit la ville et que celle-ci organise et vivifie. Ainsi le pays est finalement, en dépit de ce que les oppositions très marquées pourraient suggérer, une création indirecte de la ville. D'éventuelles inerties ou égoïsmes urbains ne contredisent pas, à ce qu'il nous semble, ce que nous venons de dire.

Ce schéma est valable pour beaucoup de pays de la zone méditerranéenne et dans de nombreuses phases de l'histoire. La Grèce centrale et le Péloponnèse nous apparaissent, dans la période antique, comme une véritable mosaïque de contrées (comarques, nous dirions en catalan), chacune avec sa ville respective. C'est exactement ce qui se produit, mais à l'époque médiévale, dans les péninsules italique et ibérique. Dans certains cas, l'ensemble ville-pays assume de telles prérogatives militaires, politiques et administratives, qu'il se convertit en ville-état. Là encore, de nombreux exemples de la Grèce classique, entre autres, viennent á l'esprit immédiatement.

De la même façon, la ville d'Iviça apparaît comme un cas parfait de ville-pays après la Reconquête. Peut-étre aussi le noyau d'Iviça a-t-il présenté ce caractère, comme nous l'avons suggéré précédemment, dans l'antiquité. Mais il n'y a aucun doute en ce qui concerne les siècles du moyen-âge, comme on va le voir dans l'analyse de ses fonctions, en ajoutant cet élément original que l'influence d'Iviça coïncide avec les limites extrêmes de l'île. La contrée dépendante d'Iviça est en effet, on l'a vu, l'île tout entière. Sans doute la coïncidence et même la confusion - unique dans les Baléares et rare dans beaucoup d'îles de la Méditerranée - entre le toponyme désignant la ville, d'une part, et l'ensemble de I'Ile, d'autre part, sont-elles plus significatives qu'il ne semble.

Plus encore : pendant la période traditionnelle, on pourrait presque dire qu'Iviça est une ville-état. Un historien a souligné ce fait récemment (ESCANDELL, 1957). L'insularité est accentuée, pendant ces siècles, par une remarquable indépendance militaire, politique, administrative et économique. Cette indépendance augmente, en fait, à cause des attaques venues de l'extérieur, en particulier de celles des pirates barbaresques.

\section{La fonction religieuse}

Que represente la ville d'Iviça, pendant la période traditionnelle, pour le paysan?. En premier lieu un centre religieux, non seulement le plus important de l'île, mais encore, a vrai dire le seul; les autres églises sont, en fin de compte at au maximum, des sortes de délégations de celui-ci (vicaries). Nous allons préciser tout de suite cela, mais il faut dès maintenant constatar que la ville est, pout le pagès, bien plus encore: elle est la place forte, le centre politique, administratif et judiciaire, le marché, le noyau artisanal, le port.

Pendant des siècles, la ville détient la seule paroisse de l'île. L'emplacement de l'édifice de l'église sur le point le plus haut de l'agglomération urbaine, au coeur de la zone fortifiée, à côté du château, constitue une sorte de preuve tangible de son absolue hégémonie religieuse. Il existait bien quelques petites églises dans la campagne, mais il s'agissait simplement de chapelles où ne se célébrait qu'un culte temporaire. Et ce n'est que dans la premère moitié du XIV ème. siècle que, l'on érigea les églises de Sant Antoni, Santa Eulària, Sant Miquel, et probablement celle de Sant Jordi; au milieu du siècle suivant celle de Jesús. Au début du XVIII siècle, on autorisa la construction de deux nouvelles églises: celle de Sant Josep et de Sant Joan Baptista, avec le même caractère que les précédentes (MACABICH, 1916). 
Ainsi, le sanctuaire urbain, pendant plus de quatre siècles, est le seul où existe un culte permanent, où est constamment réservé le Saint Sacrement, et où est toujours maintenue l'administration des sacrements. La situation commence à évoluer dans la deuxième moitié

du XVII ème. siècle, quand les cinq églises foraines deviennent vicairies, avec résidence constante de leurs officiants, encore que dépendantes de la paroisse unique. Jusqu'à la fin du siècle suivant, pendant la neuvième décennie, on ne créera pas de paroisses rurales.

\section{La fonction militaire}

La ville est le château et les murailles. Une place forte, en somme, très difficile à aborder. Les enceintes entourent, à la base et à mi-pente, la colline d'Iviça, haut de 85 mètres, et le rendent presque inexpugnable. Il l'était déjà à l'époque musulmane, et aprés la reconstruction chrétienne, avec ses trois enceintes dont nous parte la chronique de Jaume I . Beaucoup plus tard eut lieu la grandiose construction du XV ème. siècle, avec ses vastes bastions et ses murs de calcaire hauts et épais.

Il existait dans la campagnes de petites constructions militaires de vigie et de défense. Elles formaient parfois un système coordonné, comme les tours qui jalonnaient la périphérie de I'île, ou celle qui entouraient la plaine proche de la ville (Pla de Vila). Dans d'autres cas, elles pouvaient constituer des centres isolés de défense, pour la population éparse, comme cela était pour les petites églises fortifiées, celles de Sant Jordi et Santa Eulària en constituant un excellent exemple. Mais tout cela est en fin de compte accessoire. La seule forteresse capable d'abriter hommes et biens et pouvant résister à un long siège est sans aucun doute la ville. La Vila, et aussí le castell (chateau), comme ils apparaissent déjà nommés dans la «concession » de Jaume d'Aragó en 1234: "Castrum et villam de Eviça » constituent exactement la place forte de l'île.

\section{Les fonctions politiques et administratives}

Nous voulons parler à ce propos non seulement des fonctions strictement politiques mais aussi des fonctions administratives et judiciaires. Pendant tout le XIII ème. siécle, les divers pouvoirs, dans leur nouvelle expression chrétienne et occidentale, s'accumulent peu à peu, probablement de la même façon qu'à l'époque musulmane, dans la ville d'Iviça.

Comme on le sait I'île fut divisée, aprés la Reconquête, entre les trois seigneurs qui en furent les artisans. Au terme d'une série de transferts, les pouvoirs seigneuriaux finirent par échoir au Roi, à l'Archevêque de Tarragone, et á l'Archidiacre de Sant Fructuós. Leurs délégués, les governadors et les batlles (gouverneurs, baillis), résidérent toujours dans la ville. D'autre part, malgré la diversité des vassalités, les habitants d'Iviça et Formentera constituèrent un seul municipe ou « université », dont les jurés et conseillers habitèrent aussi le noyau urbain. La création du municipe d'Iviça découle de la «concession » faite en 1299 par le roi Jaume II de Majorque, qui fut, immédiatement confirmée par les autres coseigneurs ecelésiastiques (MACABICH, 1935). En dépit du fait que cette « concession » concerne tous les habitants de l'île, celle-ci est administrée en définitive presque exclusivement, commne en tant d'autres occasions, par les prud'hommes de la cité, nobles et propriétaires fonciers essentiellement, qui constituent une véritable oligarchie urbaine. Les gens de la campagne étaient représentés par un groupe de jurés - le Jurat de mà de fora (le jury de la main foraine) - mais il ne fait pas de doute que son rôle ait été subordonné.

Les juges, officiers des coseigneurs, résidaient aussi dans la ville d'Ibiça et y rendaient justice. On doit tenir compte, de plus, que l' "université", aussi bien que les 
prud'hommes, jouèrent également un rôle important dans l'exercice de la justice. En 1343 le roi Pere IV d'Aragon décréta que les juges ne pourraient rendre leurs sentences que d'accord avec les prud'hommes (MACABICH, 1935).

\section{Les fonctions économiques}

La vila, était, à tous les points de vue, le coeur économique de I'île. En effet, celleci ne compte pas de marchés intermédiaires et de centres et ports secondaires. La campagne est simplement une zone de production agricole et forestière et d'élevage. La ville est tout, en dehors de cela: marché de vente des produits précédents, centre d'approvisionnement des paysans et d'exportation pour les produits dirigés vers l'extérieur.

Nous nous trouvons en face de deux communautés aux genres de vie diamétralement opposés et de cette opposition naît leur complémentarité et leur subordination totales. La ville est un complexe social: propriétaires-fonciers (sans être latifondiaires), clercs, fonctionnaires (au sens le plus large), commerçants et marchands, artisans, pêcheurs et marins. L'artisanat qui faisait du centre urbain un petit centre manufacturier n'était pas très nombreux quoique varié: cordonniers; tailleurs; préparateurs, fileurs et tisseurs à laine (paraires et teixidors); fabricants d'espadrilles; tanneurs; teinturiers; forgerons; ciriers; potiers; savonniers; platriers; charpentiers; maçons; ouvriers des chantiers de construction navale. Tous, en définitive, avec des activités extrarurales, à l'exception du groupe des jardiniers qui cultivaient les feixes, petit secteur d'horticulture irriguée à proximité immédiate de la ville. Tous, aussi, rigidement intégrés dans un ensemble structuré en classes sociales et groupes professionnels : classe supérieure, I'ordre ou état des chevaliers nobles, représentée dans I' "Université" par le Jurat de ma major ("jury de la main majeure"); classe moyenne, l'ordre des artisans et marchands, représentés par le Jurat de ma mitjana ("jury de la main moyenne"). Artisans et marchands sont également groupés en confréries et corporations (FAJARNÉS, 1930; MACABICH, 1935).

Par contre, la campagne apparaît comme exclusivement habitée par les pagesos, cultivateurs et éleveurs, gens occupés aussi a l'exploitation des bois. Dans les débuts, un certain nombre de propriétaires fonciers a vécu également à la campagne, quoique l'absentisme semble avoir été déjà très notable. Quant à la mise en valeur des propriétés, la subordination des paysans est également nette par rapport aux propriétaires de la ville. Pendant une longue période, les paysans constituent même une classe â laquelle manquent certaines libertés: une partie de cette classe est constituée par des musulmans soumis après la Reconquete et qui, par la suite, seront remplacés par des captifs. Les documents des XIII, XIV et XV ème. siècles ne laissent pas subsister de doute á cet égard. Une donnée particulièrement concluante est fournie à ce sujet par la lettre du roi à son gouverneur Ramon Muntaner, en 1335, conservée dans les archives municipales d'Eivissa: “ ... totas las obras rusticales... se fan per mans de catius " (tous les travaux des champs... se font par mains de captifs).

Compte tenu de ces faits, on comprendra aisément la situation preponderante, économiquement et socialement, qui était celle de la ville par rapport à la campagne. On comprendra aussi la nécessité dans laquelle étaient los campagnards d'avoir recours à la ville tant pour livrer ou offrir leurs produits que pour acquérir ce qu'ils ne produisaient pas (vêtements, ustensiles de travail, etc.). Nous n'insisterons pas davantage là-dessus, mais nous voudrions, par contre préciser davantage la fonction commerciale de la ville par rapport à l'extérieur. 


\section{Le port}

L'hégémonie économique du noyau d'Iviça sur I'île était si totale que la seule production non urbaine et qui pourtant n'était pas le fait des paysans - nous voulons parler de la production du sel - dépendait cependant exclusivement de l' "Université" d'Iviça. Nous avons deja parlé ailleurs et il y a peu de temps (VILÀ VALENTÍ, 1961) de l'importance de ce produit. Ainsi nous soulignerons seulement ici combien cette profitable activité était fonction du noyau urbain et comment son exportation s'effectuait, précisément, par son intermédiaire. Cela nous amène à parler, avec quelque précision, de la fonction portuaire.

De fait, la ville d'Iviça n'était pas seulement le marché d'échanges et le centre artisanal de l'île. C'était aussi le port; le seul port de l'île. Il existait, c'est vrai, de petits mouillages sur toute la périphérie de l'île, en particulier dans les golfes de la côte septentrionale (baie de Sant Antoni ou Portmany, cala d'Albarca, etc.). Mais il ne s'agissait à l'exception du Portmany, que de criques d'accostage utilisées par les pécheurs, et n'ayant pas régulièrement une autre utilité. Le seul port commercial était celui du noyau urbain. Le port est installé dans le petit golfe naturel qui s'ouvre entre l'île Grossa et la pointe de la Marloca. La baie, dominée par la colline d'Iviça est assez bien abritée contre les vents; de plus, il existait déjá au moyen-âge une sorte d'appontement ou de digue de bois qui la protégeait des grains du Levant et du Sud.

Durant la période traditionnelle les entrées se réduisent a peu prés aux produits alimentaires, le blé en particulier; les sorties aux produits forestiers (bois de construction, de cháuffage, charbon de bois) et sel. Ainsi donc, l'approvisionnement de la ville et de l'île entière était complété grâce au port d'Iviça d'une part, tandis que d'autre part on exportait les produits des salines et des bois. L'importance réduite des produits agricoles aux sorties est à remarquer; elle s'explique par une économie rurale fermée et par une maigre production, de sorte que les paysans et la ville devaient consommer à peu près tout ce qui avait été récolté. En ce qui concerne, par contre, l'importance des produits forestiers, celleci ne fait aucun doute. L'auteur musulman Al Makkari, par exemple, précise déjà concrétement que "Iviça approvisionnait une grande partie de l'Afrique en bois de chauffage et sel" et que "la principale industrie de ses habitants consiste en fabriquer du charbon". Au cours des siècles qui suivent la Reconquête, la documentation montre l'importance de ce commerce. En 1299 on autorise à nouveau l'exportation du bois de construction et du charbon de bois. On menace de graves sanctions, dans les capitulations de I'"université" d'Iviça de 1454, ceux qui essaieraient de vendre à l'extérieur des bois de construction ou de chauffage sans l'autorisation voulue.

Le port d'Iviça joue donc, pendant des siècles, un rôle fondamental dans l'approvisionnement et le commerce de la ville et de la campagne. Rôle exclusif et total, de plus, puisque comme on l'a vu il n'existe aucun autre point d'embarquement et que son hinterland coincide avec l'île tout entière.

\section{L'évolution récente}

Les relations entre la ville et la campagne d'Iviça se modifient lentement à partir du XVIIIème. siècle. D'une part le noyau urbain évolue dans sa personnalité et dans sa structure interne; certaines de ses fonctions connaissent des changements fondamentaux. D'autre part, la campagne a également évolué de façon importante. Le résultat de ce nouvel état de choses est la perte, à plusieurs égards, par la ville, de son ancienne hégémonie sur la campagne. Cette dernière, de son côté, n'a pas seulement acquis une individualité 
religieuse et administrative, mais elle a également acquis une complexité sociale et économique qu'elle n'avait pas eu dans le passé. On ne pourrait plus du tout parler actuellement du noyau d'Ibiça comme d'une cité-état; mais même en tant que ville-pays, sa vie de relations a perdu de sa simplicité traditionnelle, tandis que cette vie se présente sous une forme notablement différente de celle qui existait autrefois.

\section{L'évolution de la ville}

En dépit des changements, de nombreux traits subsistent évidemment encore de la ville médiévale, ne serait-ce que du point de vue de sa physionomie et du paysage urbain qu'elle constitue. L'importance du développement d'une industrie moderne a été bien trop réduite ici pour qu'une continuité n'ait pas lieu avec le passé. Comme beaucoup de centres urbains méditerranéens, elle n'a pas offert l'image d'une rupture marquée avec le passé, comme c'est le cas des noyaux industrialisés de l'Europe occidentale et centrale. Mais l'évolution historique générale, avec ses répereussions de toutes sortes, n'a pas manqué d'avoir un réel retentissement sur la ville, encore qu'indirect et tardif.

La transformation a été décisive sur les plans politique, administratif et religieux. Quelques étapes de cette évolution sont, chronologiquement: perte des prérogatives de l' "université " tráditionnelle, aprés la guerre de Succession, au début du XVIIIème. siècle; création de l'évêché, à la fin du même siècle; établissement de municipes indépendants dans la campagne (Santa Eulària, Sant Josep, Sant Antoni et Sant Joan), tandis que la capitale était réduite á un minuscule finage municipal. Le déclin de la ville est donc bien net dans certaines de ses fonctions. Mais l'évolution générale a également des répercussions dont les effets se précisent dans le domain économique. Ils sont même parfois très évidents comme dans le cas de la perte, par l' "université" d'Iviça, des revenus des salines, dont la propiété passe à la couronne d'Espagne par décision de Philippe V, en 1715.

Il n'est pas nécessaire d'insister sur l'importance de ces aspects; il est bon de préciser, cependant, qu'à la création de l'évêché d'Iviça, en 1782, correspond paradoxalement une diminution des fonctions religieuses de la ville. En fait, à la suite de cette création eut lieu la division paroissiale définitive de I'île. Ainsi apparurent un bon nombre de paroisses extérieures dans la campagne d'lviça. A la fin, chaque municipe fut divisé en quatre paroisses: Santa Eulària, Nostra Senyora de Jesús, Santa Gertrudis et Sant Carles; Sant Josep, Sant Jordi, Sant Francesc de Paula et Sant Agustí; Sant Antoni Abad, Sant Rafel, Sant Mateu et Santa Agnès; Sant Joan Baptista, Sant Miquel, Sant Llorenç et Sant Vicenç. Formentera fut, de son côté, divisée en trois paroisses : Sant Francesc Xavier, Nostra Senyora del Pilar et Sant Ferran.

La ville cesse d'être, de cette façon, le seul centre religieux de l'île, la paroisse unique. Sur le plan municipal elle perd également sa qualité d'unique centre administratif, tandis que son rôle politique réel diminue. Il est vrai qu'elle remplit, en même temps, de nouvelles fonctions, ou que d'anciennes fonctions se développent: le noyau d'Iviça est par exemple, actuellement le centre scolaire et médical de l'île. Mais sur presque tous les plans, elle perd son hégémonie passée à cause de l'évolution décisive de quelques centres extérieurs.

\section{L'évolution de la campagne}

L'aire rurale, on vient de le voir, s'organise dans la deuxième moitié du XVIIIème. siècle et dans la première moitié du XIXème. en un ensemble de paroisses et communes ou municipes indépendants. Mais l'évolution n'affecte pas seulement les cadres religieux et administratifs. La campagne d'Iviça connait aussi une lente mais régulière évolution, qui va durer jusqu'à nos jours et qui finira par modifier notablement les structures 
économiques: certaines cultures arbustives prennent une importance prépondérante (caroubier, amandier); on crée et on étend les petits secteurs irrigués; l'élevage muletier prend de l'essor; les systémes de culture sont améliorés, avec une augmentation des rendements céréaliers; le régime des exploitations se modernise; un meilleur equilibre de la propriété rurale est réalisé. C'est là un ensemble, une série d'aspects qui ont déjà été abordés, plus ou moins partiellement, par quelques auteurs (FAJARNÉS C., 1961), mais qu'il conviendrait d'étudier isolèment d'une manière approfondie dans leur développement, leurs conséquences et leur structure d'ensemble. Cela donnerait lieu, à coup sûr, à une intéressante monographie, pour un historien de l'évolution économique et sociale des pays méditerranéens dans sa penultième phase, au même temps ou avant l'éclat du tourisme.

De nombreux éléments restent à élucider. Un d'entre eux, peut-être le moins envisagé et le plus intéressant, est celui de l'influence que la croissance de l'effectif humain a exercé sur l'évolution de la campagne. Dans quels domaines, l'augmentation de la population rurale d'Ivlça, fait évident depuis presque deux siècles, joue-t-elle un rôle. Car elle détermine, par exemple, le début d'une phase de forte émigration insulaire, aspect que nous ne pouvons traiter maintenant. Ce que nous devons souligner ici, c'est que cette augmentation apparait comme un facteur de base dans tous les éléments en cours de transformation dans l'aire campagnarde. C'est elle qui a nourri, sur un plan strictement démographique, la forte émigration qui s'est produite de la campagne vers la ville, avec une forte recrudescence de la tendance séculaire, au début du XIXème. siècle (pétitions et dispositions restrictives de l'administration municipale d'Iviça en 1813 et 1832). Ce fait contribue à créer, d'une part, une opposition plus vive encore entre le noyau urbain et la campagne.

Tout cela exerce une influence sur le peuplement rural. Autour de quelques centres paroissiaux apparait peu à peu un habitat concentré, formé exclusivement de pagesos, comme par exemple á Sant Joan et Sant Miquel, ou de pagesos et de pêcheürs, comme à Sant Antoni et Santa Eulalia. En 1845, selon le rapport du chef politique des Baléares, «il n'y a d'autre population véritablement agglomérée que celle de la capitale, quelques cinquante maisons à Santa Eulària, et un peu plus de vingt dans le port de Sant Antoni ». Mais cent ans ne s'étaient pas écoulés que la population concentrée montrait dejà une notable augmentation: en 1930, quelque 700 habitants étaient agglomérés dans le noyau de Sant Antoni, quelques 350 à Santa Eulària, et une centaine à Sant Joan. La dispersion continue à predominer nettement, mais la nouvelle tendance, qui deviendra encore plus évidente au cours des trente dernières années, se dessine déjà nettement. L'amélioration des communications, qui se réalise á partir des dernières décennies du siècle passé, a exercé sans doute son influence à ce sujet.

L'évolution économique de la campagne a des répercussions, évidemment, sur le mouvement du port d'Iviça. En 1845, selon le rapport précité, il était encore conforme aux tendances traditionnelles: importation d'aliments et de draps, exportation de produits forestiers (bois de chauffage et de construction, charbon de bois, écorces de pin) et sel. Mais les produits forestiers diminuent régulièrement jusqu'à nos jours, à l'exception du bois de construction, tandis que les importations se diversifient et que les sorties de produits agricoles prennent de l'importance: caroubes, amandes, céréales, produits avicoles, fruits et produits horticoles (MOLL, 1961).

\section{Situation actuelle et perspectives}

L'insularité d'Iviça garde toute son importance, de nos jours, et l'isolement qui en resulte continue d'entraîner dans I'île une évolution humaine originale. Dans le domaine religieux, politique et militaire, cette insularité est encore cause, comme par le passé, de la 
présence, à Iviça, d'un évèque, tandis, que l'Etat moderne y a son délégué de gouvernement, son commandant militaire et son juge de première instance. Mais le fait fondamental reste que contrairement à ce qui se produisait dans le passé, l'insularité d'Iviça n'entraîne plus une hégémonie totale de la ville sur le reste de I'île. Au contraire, la ville a perdu certains privilèges et prérogatives, tandis que les campagnes s'organisaient et que des noyaux de population groupée apparaissaient et progressaient. En somme, on peut parler, depuis quelques dizaines d'années, d'un réveil des campagnes d'Iviça.

Un des facteurs qui peuvent augmenter le réveil campagnard est la revalorisation de certains produits agricoles. Il s'agit parfois d'un fait accidentel, comme dans le cas des années après la guerre civile; mais cet essor peut devenir définitif s'il se fonde sur des bases plus solides (meilleures techniques dans les cultures traditionnelles, accroissements des surfaces irriguées, essor des produits laitiers et avicoles, et des cultures de fruits et primeurs). Il faut tenir compte, par exemple, que pour l'année 1960 la vente de la pomme de terre précoce insulaire a atteint la váleur, selon les calculs paraissant les plus conformes à la réalité, de quelques 40 millions de pesetas.

Un autre facteur de progrès est le tourisme. Après le remarquable développement pris par ce dernier au cours des dix années passées, avec des répercussions sociales et économiques à la fois, il est devenu un élément fondamental de l'économie d'Iviça. On calcule qu'en 1960, quelque 30.000 touristes espagnols et étrangers - ces derniers particuliérement nombreux - sont arrivés dans I'île, ce qui represente au mnimum un apport de 92 millions de pesetas (JOTAZOR, 1961). Cette année (1962) les deux chiffres ont été, selon toute vraissemblance, amplement dépassés. Que peut représenter cette activité économique par rapport au problème qui nous occupe?. Sans aucun doute un accroissement de la prospérité générale de I'île tout entière. S'il est vrai, en effet, que ce tourisme est en partie localisé dans la ville elle-méme, il concerne aussi de façon importante, deux autres centres (Sant Antoni, surtout, et Santa Eulària), quelques secteurs de la périphérie insulaire et, indirectement, en définitive, tout le monde rural. En dépit du fait que c'est la ville d'Iviça qui canalise tout le mouvement touristique, maritime et aérien, on peut dire en toute objectivité qu'aujourd'hui l'île a deux capitales touristiques : Iviça même et Sant Antoni. Le développement de ce dernier centre, qui mériterait une étude approfondie, a été, en effet, impressionnant. Avec sa magnifique baie - le "Portus Magnus" des Romains, le Portmany des Catalans -, entouré d'excellentes plages et doté d'un bon équipement hotelier (onze hôtels, vingt neuf pensions, et de nombreux appartements de location, ce qui dépasse largement l'équipement hôtelier de la ville d'Iviça) et avec son nouveau port, Sant Antoni est dans de bonnes conditions pour continuer sa favorable évolution de grand centre d'attraction touristique des Baléares et du Levant espagnol. Aussi on peut signaler le possible rôle de Santa Eulària.

Qu'en est-il des perspectivas futures?. Celles-ci doivent comporter - outre le développement d'autres activités économiques -, une certaine industrialisation. Industrialisation, en particulier, dans des domaines plus stables que le tourisme (l'irrigation et le tourisme ne sont-ils pas en train de devenir deux véritables mythes dans l'esprit de beaucoup d'espagnols?) et plus diversifiée et avancée qu'un simple conditionnement des produits ruraux. Comment ce fait se traduirait-il dans les rapports entre ville et campagne? Ce problème et les possibles répercussions économiques et sociales d'un développement industriel méritent qu'on y prête attention. Nous pensons, par exemple au phénoméne d'une forte inmigration qui pourrait se produire, consécutive à une industrialisation ou à un fort dévéloppement du tourissme. 
Quoi qu'il en soit, les rapports entre ville et campagne ont pris et prendront une forme que l'on n'aurait pas soupçonnée il y a à peine deux siècles, même au premier tiers de nôtre siècle. Il nous a semblé que le noyau urbain d'Iviça gardera naturellement une fonction stimulante et organisatrice dans l'avenir de I'île, mais cela seulement si la ville sait se montrer à la hauteur du rôle qu'elle peut jouer. Enfin nous pouvons bien prévoir un équilibre futur basé sur de nouvelles et originales données, qui verra enfin se réaliser dans l'île une véritable harmonie économique et sociale entre ville et campagne.

Institut de Géographie. Université de Murcie. Décembre, 1961.

\section{Bibliographie citée}

ESCANDELL BONET, BARTOLOMÉ (1957) : Entorn a la creació de la ciutat cristiana d’Eivissa (1235).In "Extret del "Llibre d'Eivissa" de VILLANGÓMEZ, v. cit., Barcelona, Gràfiques Diamant. Biblioteca Selecta.

FAJARNÉS CARDONA, ENRIQUE (1961) : Ibiza en los siglos XIX y XX. In "Boletín de la Camara Oficial de Comercio, Industria y Navegación de Palma de Mallorca". Palma. LXI, 630. 20-26.

FAJARNÉS TUR, ENRIQUE (1930) : Política económica de Ibiza en el siglo XVI. Palma, Imprenta Hija de J. Colomar.

JOTAZOR (ZORNOZA BERNABEU, JOSÉ) (1961) : El turismo en Ibiza y Formentera. In "Boletín de la Camara Oficial de Comercio, Industria y Navegación de Palma de Mallorca". Palma. LXI, 630. 48-54.

MACABICH LLOBET, ISIDORO (1915) : Santa María la Mayor. Los cronistas. (Apuntes Históricos). Eivissa, Imprenta de Mariano Tur, XX+152.

MACABICH LLOBET, ISIDORO (1935) : Historia de Ibiza, II. El Feudalismo. Palma, Imp. de Francisco Soler Prats, 78.

MACABICH LLOBET, ISIDORO (1942) : Historia de Ibiza, VI. Crónicas siglo XVII. Palma, Imp. de la Viuda de Francisco Soler Prats, 45.

MACABICH LLOBET, ISIDORO (1943) : Historia de Ibiza, VII. Crónicas siglo XVIII. Palma, Imp. De la Viuda de Francisco Soler Prats, 62.

MACABICH LLOBET, ISIDORO (1955) : Historia de Ibiza, VIII. Crónicas del siglo XIX. Palma

MOLL MARQUÉS, FRANCESC DE B. (1961) : El puerto de Ibiza y su comercio. In "Boletín de la Camara Oficial de Comercio, Industria y Navegación de Palma de Mallorca". Any LXI, n. 630. 65-84

QUADRADO NIETO, JOSÉ MARÍA (1847) : Forenses y ciudadanos. Historia de las disenciones civiles de Mallorca en el siglo XVI. Palma, Esteban Trias, Hi ha un edició de 1895 feta a Palma per Amengual y Muntaner.

VILÀ VALENTÍ, JOAN (1950) : Formentera. Estudio de geografía humana. In "Estudios Geográficos.”. CSIC, Madrid. XI, 40, 389 - 442 + VII làms + 9 gravats.

VILÀ VALENTÍ, JOAN (1961) : Las salinas de Ibiza y Formentera. In “ "Boletín de la Cámara Oficial de Comercio, Industria y Navegación de Palma de Mallorca". Any LXI, n. 630. pàgs. 35 - 41.

VILlANGÓMEZ LlOBET, M. (1957) : Llibre d’Eivissa. Paisatge. Història. Antologia. Barcelona, Biblioteca Selecta de Ciutats i Paisatges, 230+VIII làms. 